\title{
On non-hamiltonian graphs for which every vertex-deleted subgraph is traceable
}

\author{
CAROL T. ZAMFIRESCU*
}

\begin{abstract}
We call a graph $G$ a platypus if $G$ is non-hamiltonian, and for any vertex $v$ in $G$, the graph $G-v$ is traceable. Every hypohamiltonian and every hypotraceable graph is a platypus, but there exist platypuses which are neither hypohamiltonian nor hypotraceable. Among other things, we give a sharp lower bound on the size of a platypus depending on its order, draw connections to other families of graphs, and solve two open problems of Wiener. We also prove that there exists a $k$-connected platypus for every $k \geq 2$.
\end{abstract}

Key Words. Non-hamiltonian; traceable; hypohamiltonian; hypotraceable

MSC 2010. 05C38, 05C45

\section{Introduction}

Throughout this paper all graphs are undirected, finite, connected, and contain neither loops nor multiple edges, unless explicitly stated otherwise. A graph $G$ is hamiltonian (traceable) if it contains a hamiltonian cycle (hamiltonian path), i.e. a spanning cycle (spanning path). $G$ is called hypohamiltonian (hypotraceable) if $G$ is non-hamiltonian (non-traceable), but for every vertex $v$ in $G$ the graph $G-v$ is hamiltonian (traceable). See the survey [18] by Holton and Sheehan for an overview of results on hypohamiltonian graphs. Not included therein is material published in the last two decades, for instance work of Máčajová and Škoviera [22, 23], Araya and Wiener [1, 41], McKay [24] and articles by the author and his collaborators [11, $12,21,42-44]$.

*Department of Applied Mathematics, Computer Science and Statistics, Ghent University, Krijgslaan 281 - S9, 9000 Ghent, Belgium; e-mail address: czamfirescu@gmail.com 
$G$ is almost hypohamiltonian if $G$ is non-hamiltonian, there exists a vertex $w$ in $G$ such that $G-w$ is non-hamiltonian, and for each vertex $v \neq w$ in $G$ the graph $G-v$ is hamiltonian. $w$ is called the exceptional vertex of $G$. We study in this paper a new class of graphs, closely related to above three families: a graph $G$ is called a platypus - an egg-laying mammal - if $G$ is non-hamiltonian, and for any vertex $v$ in $G$ the graph $G-v$ is traceable. We will denote the family of all platypuses of connectivity $\kappa$ with $\mathcal{P}_{\kappa}$ and put $\mathcal{P}=\bigcup_{\kappa} \mathcal{P}_{\kappa}$.

Every hypohamiltonian and every hypotraceable graph is a platypus, but there exist platypuses which are neither hypohamiltonian nor hypotraceable. This was mentioned in the author's paper [43]. The potential interest this class of graphs might exhibit was suggested to the author by Kenta Ozeki in a conversation in 2012.

In order to draw connections between results proven here and Gábor Wiener's work [40] presented in Bordeaux in 2014, we require the following. Let $G$ be a graph and $\mathcal{T}(G)$ the set of all spanning trees of $G$. Denote with $\ell(T)$ the number of leaves of a tree $T$. The minimum leaf number $\operatorname{ml}(G)$ of a possibly disconnected graph $G$ is defined as

$$
\operatorname{ml}(G)=\left\{\begin{array}{cl}
\infty & \text { if } G \text { is not connected, } \\
\min _{T \in \mathcal{T}(G)} \ell(T) & \text { if } G \text { is connected but not hamiltonian, } \\
1 & \text { if } G \text { is hamiltonian. }
\end{array}\right.
$$

Consider an integer $\ell \geq 2$. A graph $G$ with $\operatorname{ml}(G)=\ell$ is called $\ell$-leaf-critical if $\operatorname{ml}(G-v)=\ell-1$ for every $v \in V(G)$, and $\ell$-leaf-stable if $\operatorname{ml}(G-v)=\ell$ for every $v \in V(G)$. The family of all 2-leaf-critical graphs (3-leaf-critical graphs) coincides with the family of all hypohamiltonian (hypotraceable) graphs. Wiener [40] shows that $\ell$-leaf-stable and $\ell$-leaf-critical graphs exist for every $\ell \geq 2$, and studies these graphs under the additional condition of planarity. Using these results, he solves affirmatively the open problem of Gargano, Hammar, Hell, Stacho, and Vaccaro [10, p. 93] whether non-traceable non-hypotraceable arachnoid graphs, defined in [10], exist. We observe that every platypus is arachnoid.

For a platypus $G$ we have $\operatorname{ml}(G) \in\{2,3\}$. It is 2 if and only if $G$ is traceable and 3 if and only if $G$ is hypotraceable. Furthermore, $\operatorname{ml}(G-v)$ in general depends on $v \in V(G)$, and is either 1 or 2 . (For any graph $G$, if $\operatorname{ml}(G)=\ell$, then $\operatorname{ml}(G-v) \geq \ell-1$ for all $v \in V(G)$. So if in a platypus $G$ we have $\operatorname{ml}(G)=3$, then necessarily $\operatorname{ml}(G-v)=2$ for all $v \in V(G)$, whence, $G$ is a 3-critical graph, i.e. hypotraceable.) $\mathcal{P}$ contains all 2-leaf-critical, 3-leaf-critical, and 2-leaf-stable graphs, and no other leaf-critical or leaf-stable graphs. But $\mathcal{P}$ is larger than the three aforementioned families - a polyhedral (i.e. planar and 3-connected) platypus not belonging to any of the three families will be discussed in Section 5 .

We now introduce the notation used throughout this article. When we speak of a cut, we always refer to a vertex-cut. Denote with $P_{k}\left(C_{k}\right)$ a path (cycle) on $k$ vertices. Let $G$ be a graph. We call a path $P \subset G$ of length at least 2 and with end-vertices $v, w$ an ear if $\{v, w\}$ is a cut in $G$ and every vertex in $V(P) \backslash\{v, w\}$ has degree 2 in $G$. An ear on $k$ vertices will be called a $k$-ear. Furthermore, we will require an ear not to contain any super-ears, i.e. for every ear $P$ there exists no ear $P^{\prime}$ such that $P \subsetneq P^{\prime}$. 
We write $v w$ for the edge between the vertices $v$ and $w$. For a path $P$ with $V(P)=\left\{a_{1}, \ldots, a_{k}\right\}, k \geq 3$, and $E(P)=\left\{a_{i} a_{i+1}\right\}_{i=1}^{k-1}$ we write $a_{1} \ldots a_{k}$. We call $v \in V(G)$ naughty if the set of vertices adjacent to $v$, the neighbourhood of $v$ denoted in the following with $N(v)$, contains (at least) two vertices each of degree 2. Put $N[v]=N(v) \cup\{v\} . \Delta(G)$ denotes the maximum degree of $G$. A vertex is cubic if it has degree 3 and a graph is cubic if all of its vertices are cubic.

A graph $G$ is bipartite if there exist disjoint sets $A, B \subset V(G)$ such that $A \cup B=$ $V(G)$, and every edge of $G$ is of the form $a b: a \in A, b \in B$. We call $(A, B)$ a bipartition of $G$. $G$ is balanced if there exists a bipartition $(A, B)$ of $G$ such that $|A|=|B|$, and unbalanced if it is not balanced. For $S \subset V(G)$, let $G[S]$ be the subgraph of $G$ induced by $S$.

Let $G$ be a graph of connectivity 2 and $X=\{v, w\}$ a cut in $G$. Let $A$ be a connected component of $G-X$, and put $H=G[A \cup\{v, w\}]$. Consider a graph $J$ and $x, y \in V(J)$. We replace $(H, v, w)$ with $(J, x, y)$ (or simply $H$ with $J$ ) if in $G-A$ and $J$ we identify $v$ with $x$ and $w$ with $y$. In case $H$ or $J$ are paths, $v, w$ and $x, y$ will be their respective leaves.

\section{Structural results}

The following proposition contains basic facts concerning platypuses. These will often be used tacitly in the remainder of this paper.

\section{Proposition 2.1.}

(i) Every platypus is 2-connected.

(ii) A platypus containing a triangle with at least one cubic vertex is traceable.

(iii) Let $G$ be a platypus containing a $k$-ear $P$, where $k \in\{3,4\}$. Then for every non-adjacent $v, w \in V(P)$ the graph $G+v w$ is a platypus.

(iv) Every bipartite platypus must be balanced.

(v) A platypus does not contain naughty vertices. In particular: no platypus contains a $k$-ear, $k \geq 5$.

(vi) If a platypus contains a vertex of degree 2 , then it is traceable.

(vii) If a platypus contains a 4-ear $H$, then $H$ can be replaced with a 3 -ear, and the resulting graph is a platypus, too.

Proof. (i) Let $v$ be a cut-vertex of a platypus $G$. Then $G-v$ cannot be traceable.

(ii) Let $T$ be a triangle with $V(T)=\left\{v_{1}, v_{2}, v_{3}\right\}$ in a platypus $G$, and let $v_{3}$ be cubic. $G-v_{1}$ contains a hamiltonian path $\mathfrak{p}$. If $v_{2} v_{3} \in E(\mathfrak{p})$, then $\left(\mathfrak{p}-v_{2} v_{3}\right) \cup v_{2} v_{1} v_{3}$ is a hamiltonian path in $G$. If $v_{2} v_{3} \notin E(\mathfrak{p})$, then $v_{3}$ is an end-vertex of $\mathfrak{p}$ and $\mathfrak{p}+v_{3} v_{1}$ shows that $G$ is traceable.

(iii) Certainly, all paths in $G$ remain intact after adding $v w$. Now assume $G+v w$ is hamiltonian, and let $x, y$ be the end-vertices of $P$. Since $\{x, y\}$ is a 2-cut of $G$, there exists a hamiltonian path in $G-(V(P) \backslash\{x, y\})$, the end-vertices of which are $x$ and $y$. But then $G$ is hamiltonian, a contradiction. 
(iv) If $(A, B)$ is a bipartition of $G$ with $|A|<|B|$, then deleting a vertex from $A$ cannot yield a traceable graph.

(v) Let $G$ be a platypus containing a naughty vertex $v$. Let $v^{\prime}, v^{\prime \prime} \in N(v)$ have degree 2. Since $G$ is a platypus, $G-v$ contains a hamiltonian path $\mathfrak{p}$. The endvertices of $\mathfrak{p}$ must be $v^{\prime}$ and $v^{\prime \prime}$. But then $\mathfrak{p} \cup v^{\prime} v v^{\prime \prime}$ is a hamiltonian cycle in $G$, a contradiction.

(vi) Let the platypus $G$ have a vertex $v$ of degree 2 . If $w \in N(v)$, then $G-w$ contains a hamiltonian path $\mathfrak{p}$ ending in $v \cdot \mathfrak{p}$ can now be extended to a hamiltonian path in $G$.

(vii) Let $G$ be the original and $G^{\prime}$ the resulting graph. Clearly $G^{\prime}$ is nonhamiltonian. Denote the vertices of $H$ which are not its end-vertices with $x$ and $y$ and let $w$ be the vertex which replaces $x$ and $y$. Any hamiltonian path in $G$ using $x y$ may now use $w$. It remains to see that $G^{\prime}-w$ is traceable. Let $\mathfrak{p}$ be a hamiltonian path in $G-x$. Now $\mathfrak{p}-y$ is a hamiltonian path in $G^{\prime}-w$.

In fact, the idea behind (vii) can be extended significantly, as we shall explore in Section 4. Applying (iii) to the graph shown in Fig. 1 (a) we obtain a planar platypus with no cubic vertices. This contrasts Thomassen's theorem [34] stating that every planar hypohamiltonian graph contains a cubic vertex - we will come back to this intriguing fact in Section 3. Since the graph from Fig. 1 (a) will appear frequently in future arguments, we will call it $\mathfrak{T}$ in the remainder of this article.

Proposition 2.2. Let $G$ be a platypus containing a triangle $v_{1} v_{2} v_{3}$ with $v_{1}, v_{2} \in$ $V(G)$ cubic. Consider vertices $w, v_{1}^{\prime}, v_{2}^{\prime}, v_{3}^{\prime} \notin V(G)$. Then

$$
\begin{gathered}
R(G)=\left(V(G) \cup\{w\}, E(G) \cup\left\{w v_{i}\right\}_{i=1}^{3}\right), \\
S(G)=\left(V(G) \cup\left\{v_{1}^{\prime}, v_{2}^{\prime}\right\}, E(G) \cup\left\{v_{1} v_{1}^{\prime}, v_{1}^{\prime} v_{2}^{\prime}, v_{2} v_{1}^{\prime}, v_{2} v_{2}^{\prime}, v_{1} v_{2}^{\prime}, v_{3} v_{1}^{\prime}\right\}\right),
\end{gathered}
$$

and

$$
T(G)=\left(V(G) \cup\left\{v_{i}^{\prime}\right\}_{i=1}^{3}, E(G) \cup\left\{v_{1}^{\prime} v_{2}^{\prime}, v_{2}^{\prime} v_{3}^{\prime}, v_{3}^{\prime} v_{1}^{\prime}\right\} \cup\left\{v_{i} v_{i}^{\prime}\right\}_{i=1}^{3}\right),
$$

are platypuses, as well. $R, S$, and T preserve planarity and 3-connectedness.

Proof. In the remainder of this proof, $G$ will be considered as a subgraph of $R(G)$, $S(G)$ or $T(G)$, depending on which operation we are studying. We first show that $R(G)$ is a platypus. Assume $R(G)$ contains a hamiltonian cycle $\mathfrak{h}$. Ignoring symmetric cases, since $w$ is cubic we have either $v_{1} w v_{2} \subset \mathfrak{h}$ or $v_{1} w v_{3} \subset \mathfrak{h}$. In both situations we can modify $\mathfrak{h}$ to a hamiltonian cycle in $G=R(G)-w$ by replacing $v_{1} w v_{2}$ with $v_{1} v_{2}$ or $v_{1} w v_{3}$ with $v_{1} v_{3}$, yielding a contradiction. Hence $R(G)$ is non-hamiltonian.

Let $v \in V(G)$ and let $\mathfrak{r}$ be a hamiltonian path in $G-v$. If $v_{1} v_{2} \in E(\mathfrak{r})$, $v_{2} v_{3} \in E(\mathfrak{r})$ or $v_{1} v_{3} \in E(\mathfrak{r})$, then $\mathfrak{r}$ can be transformed into a hamiltonian path in $R(G)-v$. If neither of these three edges occurs in $\mathfrak{r}$, then necessarily $v_{1}$ or $v_{2}$ must be an end-vertex of $\mathfrak{r}$. Then $\mathfrak{r}+v_{1} w$ or $\mathfrak{r}+v_{2} w$ is a hamiltonian path in $R(G)-v$. Since $G$ is a platypus containing a triangle which has a cubic vertex, Prop. 2.1 (ii) implies that $G=R(G)-w$ is traceable.

Now we prove that $S(G)$ is a platypus. Assume $S(G)$ contains a hamiltonian cycle and let $\mathfrak{p}$ be its intersection with $S(G)\left[v_{1}, v_{2}, v_{3}, v_{1}^{\prime}, v_{2}^{\prime}\right]$. If $\mathfrak{p}$ is disconnected, then one of its components is a path with end-vertices $v_{1}$ and $v_{2}$. Substituting $v_{1} v_{2}$ 
for this path yields a hamiltonian cycle in $G$, a contradiction. If $\mathfrak{p}$ is connected, then $\mathfrak{p}$ is a path and there are essentially two different cases. If $v_{1}$ and $v_{2}$ are the endvertices of $\mathfrak{p}$ then substituting $v_{1} v_{3} v_{2}$ for $\mathfrak{p}$ yields a hamiltonian cycle in $G$, and if $v_{1}$ and $v_{3}$ are the end-vertices of $\mathfrak{p}$ then substituting $v_{1} v_{2} v_{3}$ for $\mathfrak{p}$ yields a hamiltonian cycle in $G$. In either case, a contradiction is obtained.

Let $\mathfrak{s}$ be a hamiltonian path in $G-v$, where $v \in V(G) \backslash\left\{v_{1}, v_{2}\right\}$. Assume $v_{1} v_{2} \in E(\mathfrak{s})$. Then $\left(\mathfrak{s}-v_{1} v_{2}\right) \cup v_{1} v_{1}^{\prime} v_{2}^{\prime} v_{2}$ is a hamiltonian path in $S(G)-v$. Assume now that $v_{1} v_{2} \notin E(\mathfrak{s})$. If $v_{2}$ is an end-vertex of $\mathfrak{s}$, then $\mathfrak{s} \cup v_{2} v_{1}^{\prime} v_{2}^{\prime}$ is a hamiltonian path in $S(G)-v$. If $v_{2}$ is not an end-vertex of $\mathfrak{s}$, then $v_{3} v_{2} \in E(\mathfrak{s})$. In this case $\left(\mathfrak{s}-v_{3} v_{2}\right) \cup v_{2} v_{2}^{\prime} v_{1}^{\prime} v_{3}$ is a hamiltonian path in $S(G)-v$. The discussion for $v \in\left\{v_{1}, v_{2}\right\}$ is very similar. Consider a hamiltonian path $\mathfrak{s}^{\prime}$ in $G-v_{2}$. If $v_{1} v_{3} \in E\left(\mathfrak{s}^{\prime}\right)$, then $\left(\mathfrak{s}^{\prime}-v_{1} v_{3}\right) \cup v_{3} v_{2} v_{2}^{\prime} v_{1}$ is a hamiltonian path in $S(G)-v_{1}^{\prime}$. If $v_{1} v_{3} \notin E\left(\mathfrak{s}^{\prime}\right)$, then $v_{1}$ is an end-vertex of $\mathfrak{s}^{\prime}$. Then $\mathfrak{s}^{\prime} \cup v_{1} v_{2}^{\prime} v_{2}$ is a hamiltonian path in $S(G)-v_{1}^{\prime}$. The argument for $S(G)-v_{2}^{\prime}$ is exactly the same.

We now show that $T(G)$ is a platypus. We have shown that $R(G)$ is nonhamiltonian, and $T(G)$ is non-hamiltonian if and only if $R(G)$ is non-hamiltonian.

Consider $v \in V(G) \backslash\left\{v_{1}\right\}$ and a hamiltonian path $\mathfrak{t}$ in $G-v$. If $v_{1}$ is an endvertex of $\mathfrak{t}$, then $\mathfrak{t} \cup v_{1} v_{1}^{\prime} v_{2}^{\prime} v_{3}^{\prime}$ is a hamiltonian path in $T(G)-v$. If $v_{1}$ is not an end-vertex of $\mathfrak{t}$, then $v_{1} v_{2} \in E(\mathfrak{t})$ or $v_{1} v_{3} \in E(\mathfrak{t})$. In the former case, substitute in $\mathfrak{t}$ the path $v_{1} v_{1}^{\prime} v_{3}^{\prime} v_{2}^{\prime} v_{2}$ for the edge $v_{1} v_{2}$, and in the latter case substitute in $\mathfrak{t}$ the path $v_{1} v_{1}^{\prime} v_{2}^{\prime} v_{3}^{\prime} v_{3}$ for the edge $v_{1} v_{3}$, and we obtain a hamiltonian path in $T(G)-v$. Since we have dealt with the case $v=v_{2}$, the case $v=v_{1}$ follows directly.

A hamiltonian path in $T(G)-v_{1}^{\prime}$ can be obtained by considering the hamiltonian path $\mathfrak{t}$ in $G-v_{3}$. We have $v_{1} v_{2} \in E(\mathfrak{t})$. Substitute $v_{1} v_{3} v_{3}^{\prime} v_{2}^{\prime} v_{2}$ for $v_{1} v_{2}$ in $\mathfrak{t}$. Similarly for $T(G)-v_{2}^{\prime}$. Consider a hamiltonian path $\mathfrak{t}^{\prime}$ in $G-v_{2}$. If $v_{1}$ is an end-vertex of $\mathfrak{t}^{\prime}$, then $\mathfrak{t}^{\prime} \cup v_{1} v_{1}^{\prime} v_{2}^{\prime} v_{2}$ is a hamiltonian path in $T(G)-v_{3}^{\prime}$. If $v_{1}$ is not an end-vertex of $\mathfrak{t}^{\prime}$, then $v_{1} v_{3} \in E\left(\mathfrak{t}^{\prime}\right)$. Replacing $v_{1} v_{3}$ with $v_{1} v_{1}^{\prime} v_{2}^{\prime} v_{2} v_{3}$ yields a hamiltonian path in $T(G)-v_{3}^{\prime}$.

Bondy observed [4] that the vertices of a triangle in a hypohamiltonian graph have degree at least 4. (This is true for hypotraceable graphs, as well.) Thus, hypohamiltonian and hypotraceable graphs do not fulfil the condition from Prop. 2.2, but $\mathfrak{T}$, the graph shown in Fig. 1 (a), does satisfy it. Yet again we see that there exist platypuses which are neither hypohamiltonian nor hypotraceable.

In a possibly disconnected graph $G$, let us write $\omega(G)$ for the number of connected components of $G$. The toughness of $G$ is defined as

$$
t(G)=\min \left\{\frac{|S|}{\omega(G-S)}: S \subseteq V(G), \omega(G-S) \neq 1\right\},
$$

and the toughness of a complete graph is convened to be $\infty$. A graph $G$ is $t$-tough whenever $t \leq t(G)$. Chvátal [6] proved that every hamiltonian graph is 1-tough.

Theorem 2.3. Every platypus is 1-tough.

Proof. Consider a platypus $G$ and let $S \subset V(G)$ have cardinality $k$. Consider $v \in V(S)$. We denote with $\mathfrak{p}$ a hamiltonian path in $G-v$. The vertices in $S \backslash\{v\}$ are on $\mathfrak{p}$ and determine at most $k$ subpaths of $\mathfrak{p}$. Each such subpath visits exactly 
one component of $G-S$ if it has more than two vertices, and none if it has just two vertices. Hence $G-S$ has at most $k$ components.

Every hamiltonian graph is a 1-tough graph which is not a platypus. Nonhamiltonian 1-tough graphs which are not platypuses are harder to describe: an example of such a graph is Nishizeki's planar triangulation from [26, Fig. 3], where it is easily verified that removing the vertex $x_{4}\left(x_{4}\right.$ as shown in [26, Fig. 3]) yields a non-traceable graph. Note that Theorem 2.3 implies that hypohamiltonian and hypotraceable graphs are 1-tough.

Let $G$ be a graph, consider its Cartesian product with $P_{2}, G \square P_{2}$, and replace each copy of $P_{2}$ with $P_{3}$. We will call the resulting graph the dotted prism over $G$ and denote it with $\dot{G}$.

\section{Theorem 2.4.}

(i) The dotted prism over a hamiltonian graph $G$ of odd order $n \geq 3$ is a platypus.

(ii) Let $G$ be a hamiltonian graph of even order containing an edge e which lies on all hamiltonian cycles occurring in $G$. Let $v^{\prime} w^{\prime}=e^{\prime}$ and $v^{\prime \prime} w^{\prime \prime}=e^{\prime \prime}$ be the two copies of e in $\dot{G}$. Then $H=\dot{G}-e^{\prime}-e^{\prime \prime}+v^{\prime} w^{\prime \prime}+v^{\prime \prime} w^{\prime}$ is a platypus.

Proof. (i) Denote the two copies of $G$ in $\dot{G}$ with $G^{\prime}$ and $G^{\prime \prime}$. Assume $\dot{G}$ is hamiltonian. Every copy of $P_{3}$ must be traversed. Since there is an odd number of copies of $P_{3}$, either we begin in $G^{\prime}$ and end up in $G^{\prime \prime}$ or vice-versa. In both cases we obtain a contradiction, so $\dot{G}$ is non-hamiltonian.

Denote the end-vertices of the $n$ copies of $P_{3}$ with $v_{i}^{\prime}$ and $v_{i}^{\prime \prime}$, where $v_{i}^{\prime} \in V\left(G^{\prime}\right)$ and $v_{i}^{\prime \prime} \in V\left(G^{\prime \prime}\right), i \in\{1, \ldots, n\}$. Call $w_{i}$ the vertex with neighbourhood $\left\{v_{i}^{\prime}, v_{i}^{\prime \prime}\right\}$ and put $W=\left\{w_{i}\right\}_{i=1}^{n}$. We now show that there exists a hamiltonian path in $H=G-w_{i}$ with $i$ arbitrary, but fixed. Since $G$ is hamiltonian, $H$ contains a cycle $\mathfrak{c}$ which visits all vertices of $H$ with the exception of $v_{i}^{\prime}$. Let $x \in N\left(v_{i}^{\prime}\right) \backslash\left\{w_{i}\right\}$ and $\{y\}=N(x) \cap W$. Then $(\mathfrak{c}-x y)+x v_{i}^{\prime}$ is a hamiltonian path in $H$.

Let $v \in V(\dot{G}) \backslash W$. W.l.o.g. $v \in V\left(G^{\prime}\right)$, so there is an integer $j$ such that $v=v_{j}^{\prime}$. As before, $\dot{G}-v_{j}^{\prime}-w_{j}$ contains a hamiltonian cycle $\mathfrak{h}$. Let $z \in N\left(v_{j}^{\prime \prime}\right) \cap V(\mathfrak{h})$. Then $\left(\mathfrak{h}-z v_{j}^{\prime \prime}\right)+v_{j}^{\prime \prime} w_{j}$ is a hamiltonian path in $\dot{G}-v_{j}^{\prime}$.

(ii) Assume $H$ has a hamiltonian cycle $\mathfrak{h}$. Due to the condition that $G$ contains an edge $e$ belonging to all hamiltonian cycles in $G, v^{\prime} w^{\prime \prime}$ and $v^{\prime \prime} w^{\prime}$ are contained in $\mathfrak{h}$. (Since if there was a hamiltonian cycle in $H$ not using the edges $v^{\prime} w^{\prime \prime}$ and $v^{\prime \prime} w^{\prime}$, we would immediately obtain a hamiltonian cycle in $G$ which would not contain $e$.) As $\mathfrak{h}$ is a hamiltonian cycle, the 3 -ear connecting $v^{\prime}$ and $v^{\prime \prime}$ and the one connecting $w^{\prime}$ and $w^{\prime \prime}$ must be traversed by $\mathfrak{h}$. Then $\mathfrak{h}$ contains only six vertices, a contradiction.

Showing that $H-v$ is traceable for every $v \in V(H)$ is very similar to the proof given in (i).

Graphs obtained from $G$ in the same manner as $H$ in the statement of Theorem 2.4 (ii) will be called modified dotted prisms of $G$ and will be denoted with $G^{\times}$. We present two consequences of Theorem 2.4, the first of which follows directly from Theorem 2.4 (ii). 
Corollary 2.5. There exist infinitely many balanced bipartite platypuses.

Using a computer, Van Cleemput [37] determined that there exist no platypuses on fewer than 9 vertices. His program also determined that there are exactly four such graphs on 9 vertices (Van Cleemput verified these results using two independent implementations): they are $\mathfrak{T}$, i.e. the graph from Fig. 1 (a), and the three graphs obtained by applying Prop. 2.1 (iii) to one, two, or three 3-ears in $\mathfrak{T}$. For order 10, the situation changes dramatically and there are many platypuses: at least forty, as communicated by Van Cleemput [37].

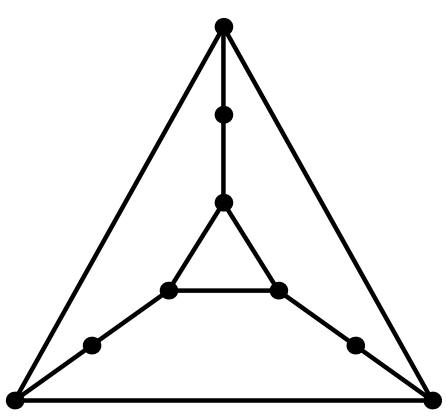

(a)

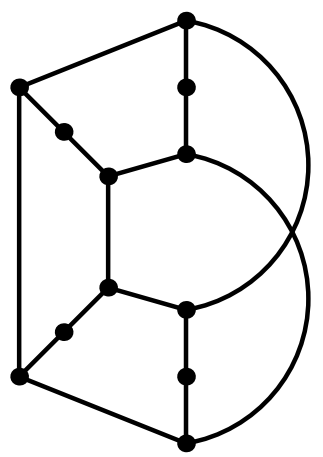

(b)

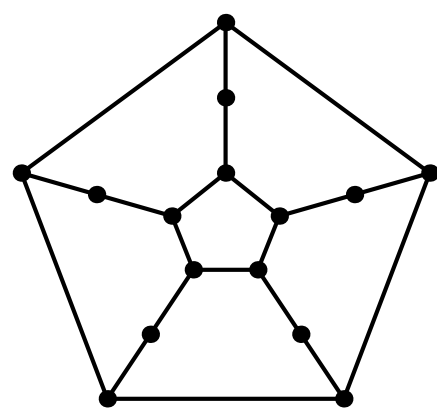

(c)

Fig. 1: (a) shows a platypus on 9 vertices, in this paper called $\mathfrak{T}$, while (b) and (c) present platypuses of order 12 and 15, resp.

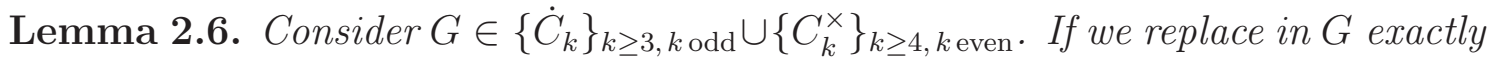
$\ell$ 3-ears with 4 -ears, $0 \leq \ell \leq k$, then the graph we obtain is a platypus.

Proof. We prove the statement by induction. $G$ is a platypus due to Theorem 2.4. Now assume we have replaced in $G$ exactly $p<k 3$-ears with 4 -ears. We denote the resulting platypus with $G^{\prime}$. In $G^{\prime}$, replace a 3-ear $u v w$ with a 4 -ear $u v v^{\prime} w$ and denote the graph we obtain with $G^{\prime \prime}$. Any hamiltonian cycle in $G^{\prime \prime}$ would use the path $u v v^{\prime} w$, which if replaced with $u v w$ would imply the hamiltonicity of $G^{\prime}$, a contradiction.

Since $G^{\prime}-u$ and $G^{\prime}-w$ are traceable, so are $G^{\prime \prime}-u$ and $G^{\prime \prime}-w$. It remains to show that $G^{\prime \prime}-v$ is traceable. Arguing as in the proof of Theorem 2.4 (since it makes no difference whether we must traverse 3-ears or 4-ears), there exists a hamiltonian path in $G-v$ which has $w$ as one of its end-vertices. Now consider this path in $G^{\prime}$ and add the edge $w v^{\prime}$. The argument for $G^{\prime}-v^{\prime}$ is exactly the same.

Corollary 2.7 (Van Cleemput and Zamfirescu). There exists a platypus of order $n$ for every $n \geq 9$, there are no platypuses on fewer vertices, and there exist exactly four platypuses on 9 vertices.

Proof. Adding to the arguments preceding Lemma 2.6, we consider the dotted prism $\dot{C}_{k}$ over $C_{k}$ for $k \geq 3$ odd and the modified dotted prism $C_{k}^{\times}$over $C_{k}$ for $k \geq 4$ even. (Fig. 1 shows (a) $\dot{C}_{3}$, (b) $C_{4}^{\times}$, and (c) $\dot{C}_{5}$.) Since we can replace in each of the graphs one or two 3-ears with 4-ears (see Lemma 2.6), we have covered all orders. 
We briefly comment upon the graphs shown in Fig. 1. Graph (b) was already given by T. Zamfirescu, see [29, Fig. 19 (a)], who asked for the largest integer $c$ such that any $c$ longest cycles of any 2-connected graph have a common vertex. (This is in the same spirit as Gallai's famous question from 1966 whether in every graph the intersection of all longest paths is non-empty [9]. Walther [38] showed that this is not the case.) Graph (b) proves $c \leq 7$. Jendrol' and Skupień [20] showed $c \leq 6$, and this is the best bound that is known. Graph (c) was already given by Thomassen, see [29, Fig. 16], to improve the bound for the smallest order of a planar 2-connected graph in which every vertex is missed by a longest cycle. Brinkmann and Van Cleemput [2] showed that Thomassen's example is the graph of smallest order with this property.

Herz [16] defines the cyclability of a graph $G$ as the greatest integer $k$ such that for every set $S \subset V(G)$ of cardinality $k$ there exists a cycle in $G$ containing $S$. The family of all graphs $G$ of cyclability $|V(G)|$ are all hamiltonian graphs, and cyclability $|V(G)|-1$ coincides with hypohamiltonicity. Cyclability has been studied extensively, for instance by Chvátal [5], Halin [15], and Holton, McKay, Plummer, and Thomassen [17].

Theorem 2.8. For every integer $t \geq 2$ there exists a platypus of cyclability $t$.

Proof. Consider $\dot{C}_{k}$ and $C_{k}^{\times}$as in the proof of Corollary 2.7. Denote with $W_{k}$ the set of vertices added to the copies of $P_{2}$ when constructing the dotted or modified dotted prism. We have $\left|W_{k}\right|=k$. Since $\dot{C}_{k}$ and $C_{k}^{\times}$are non-hamiltonian, they have no cycle containing $W_{k}$, so their cyclability is at most $k-1$. Consider $G \in$

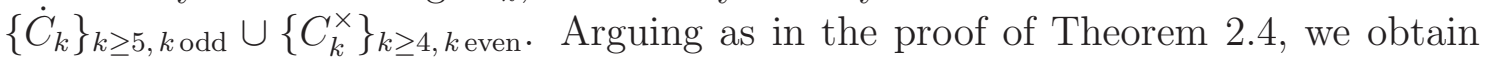
the following.

Claim. The circumference of $G$ is $|V(G)|-2=3 k-2$. Let $P=$ uvw be a 3-ear in $G$. Then there exists in $G$ a longest cycle avoiding $u$ and $v$.

It remains to show that for any set $S$ of $k-1$ vertices in $G$, there is a cycle containing $S$. $G$ contains at least one 3-ear $P$ for which $V(P) \cap S=\emptyset$, since $|S|=k-1$. Using the Claim, we are done.

Finally, the circumference of $\dot{C}_{3}$ is $\left|V\left(\dot{C}_{3}\right)\right|-1=8$, see Fig. 1 (a). In the case of $\dot{C}_{3}$ we are now finished as well, as in a 2-connected graph any two vertices lie in a cycle.

Theorem 2.9. Let $G$ be a platypus of order $n$ and size $m$. We have $m \geq\lceil 5 n / 4\rceil$, and this bound is sharp.

Proof. We claim that at most half of the vertices of $G$ have degree 2. Assume the contrary, i.e. that $G$ has $p<n / 2$ vertices of degree greater than 2. By Prop. 2.1 (v), $G$ contains no naughty vertices, which implies that every vertex of degree $\neq 2$ has either no neighbours of degree 2 or one neighbour of degree 2 , and that every vertex of degree 2 has at least one neighbour of degree greater than 2 . Therefore, the number of vertices of degree 2 is at most $p$. But then $G$ has in total at most $2 p<n$ vertices, a contradiction. This immediately yields that $2 m \geq 2 n / 2+3 n / 2=5 n / 2$, which gives the advertised bound. 
Consider $G \in\left\{\dot{C}_{k}\right\}_{k \geq 3, k \text { odd }} \cup\left\{C_{k}^{\times}\right\}_{k \geq 4, k \text { even. }}$ By replacing in $G$ exactly $\ell$ 3-ears with 4 -ears, $0 \leq \ell \leq k$, we have constructed graphs which prove the sharpness of the bound. That these graphs are indeed platypuses follows from Lemma 2.6.

An immediate consequence of Corollary 2.7 and Theorem 2.9 is that $\mathfrak{T}$ is the smallest platypus both in terms of order and size.

\section{Links to other families of graphs}

The first important distinction to be made is between traceable and non-traceable platypuses. While the latter coincides with the family of all hypotraceable graphs, the former contains (but does not coincide with) the family of all hypohamiltonian graphs.

A snark is a cyclically 4-edge-connected cubic graph with chromatic index 4 and girth at least 5. Isaacs [19] showed that there exist infinitely many snarks. Fiorini [8] was the first to prove explicitly that there exist infinitely many hypohamiltonian snarks. (Implicitly, and earlier, this had already been done by Gutt [14].) However, Fiorini's proof is incorrect. An erratum and an extension of this theorem can be found in $[11,12]$. Since every hypohamiltonian graph is a platypus, on one hand we have that there are infinitely many snarky platypuses (i.e. graphs which are both a snark and a platypus). On the other hand, Tait [30] proved that the Four Colour Theorem is equivalent to the statement that no snark is planar. As there exist infinitely many planar hypohamiltonian graphs [33], there exist infinitely many non-snarky platypuses.

Let $G$ be a graph containing a 4-cycle $v_{1} v_{2} v_{3} v_{4}=C$. We denote with $\operatorname{Th}\left(G_{C}\right)$ the graph obtained from $G$ by deleting the edges $v_{1} v_{2}$ and $v_{3} v_{4}$ and adding a 4-cycle $v_{1}^{\prime} v_{2}^{\prime} v_{3}^{\prime} v_{4}^{\prime}$ disjoint from $G$, and the edges $v_{i} v_{i}^{\prime}, 1 \leq i \leq 4$, to $G$. This operation was introduced by Thomassen [35].

Consider the almost hypohamiltonian graph $G$ constructed by the author in [43], see Fig. 2, and denote the exceptional vertex of $G$ with $w$. It is easy to verify that $G-w$ is traceable. (Note that a priori, in an almost hypohamiltonian graph $G$ with exceptional vertex $w$, the graph $G-w$ may be non-traceable; see the next paragraph.) As $G$ is almost hypohamiltonian, for every $v \in V(G) \backslash\{w\}$ the graph $G-v$ is hamiltonian, so certainly traceable. This property is not lost if an infinite family is constructed by applying to $G$ the operation $\mathrm{Th}$-for details, see [43, Theorem 1]. Thus, we have shown that there exist infinitely many almost hypohamiltonian platypuses. (Here we have sketched a proof of the fact that there exist infinitely many polyhedral platypuses. A rigorous treatment is given in Section 5.)

Let $H$ be a hypotraceable graph and $w \notin V(H)$ a vertex. Construct a graph $G$ by joining $w$ with all vertices of $H$. G is almost hypohamiltonian with exceptional vertex $w$, yet not a platypus, as $G-w=H$ is non-traceable. As there exist infinitely many hypotraceable graphs [31], we have shown that there are infinitely many almost hypohamiltonian graphs which are not platypuses. This holds viceversa as well, since every hypotraceable graph is a platypus, and no graph can be both hypotraceable and almost hypohamiltonian. Furthermore, Wiener [40] showed recently that if $G$ is a hypotraceable graph with a cut $\{a, b\}$, then $G+a b$ is 2-leafstable, and thus a traceable platypus. Related to this, see Prop. 3.1 (ii). 


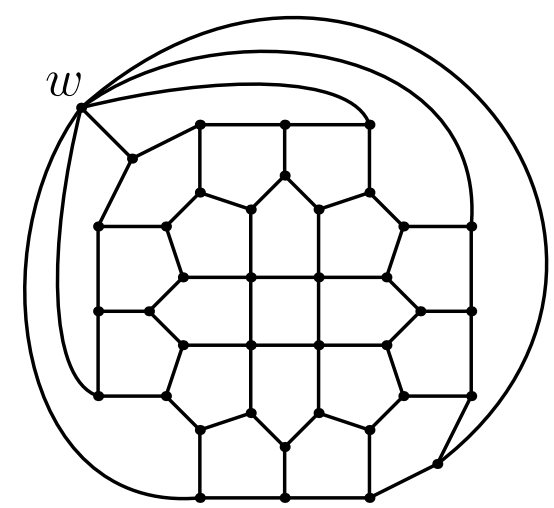

Fig. 2: A polyhedral almost hypohamiltonian graph with exceptional vertex $w$.

A further motivation for introducing platypuses follows. Chvátal [4] conjectured that if $G$ is hypohamiltonian, and $e \in E(G)$ an edge between vertices each of degree at least 4, then $G-e$ is hypohamiltonian, too. Although Chvátal's conjecture is not true as shown by Thomassen [32] (and even has planar counterexamples as shown by the author [43]), the following proposition does hold.

\section{Proposition 3.1.}

(i) Let $G$ be a hypohamiltonian graph. For any $e \in E(G)$, the graph $G-e$ is a platypus.

(ii) Let $H$ be hypotraceable graph, and $v, w \in V(H)$ non-adjacent. Then $H+v w$ is a platypus.

Proof. (i) Put $G^{\prime}=G-e$. $G$ is non-hamiltonian, so $G^{\prime}$ is, too. Consider $v \in V\left(G^{\prime}\right)$. Since $G$ is hypohamiltonian, there exists a hamiltonian cycle in $G-v$, so there exists a hamiltonian path in $G^{\prime}-v$.

(ii) Put $H^{\prime}=H+v w$. Since for every $u \in V(H)$ there is a hamiltonian path in $H-u$, this evidently also holds for $H^{\prime}$. Assume $H^{\prime}$ were hamiltonian. Then $H^{\prime}-v w=H$ would be traceable, a contradiction.

Neither (i) nor (ii) can be inverted: (ii) is obvious, and concerning (i), consider a traceable platypus. Adding an edge between the end-vertices of a hamiltonian path would yield a hamiltonian graph. Indeed, for a platypus $G$ and a pair of nonadjacent vertices $v$ and $w$ in $G$, the graph $G+v w$ is a platypus if and only if there exists no hamiltonian path (in $G$ ) between $v$ and $w$. It may prove interesting to consider in future work "full" platypuses, i.e. platypuses to which no further edge may be added without losing the property of being a platypus.

The first part of the following proposition was mentioned, but not proven, in the author's paper [43], while the second part is a special case of [43, Lemma 1] (re-proven here for convenience), since a platypus is non-traceable if and only if it is hypotraceable. 


\section{Proposition 3.2.}

(i) An almost hypohamiltonian graph $G$ minus its exceptional vertex $w$ is a platypus.

(ii) The join $H^{\prime}$ of a non-traceable platypus $H$ and $K_{1}=(\{w\}, \emptyset)$ is almost hypohamiltonian with exceptional vertex $w$.

Proof. (i) Put $G^{\prime}=G-w$. By definition, $G^{\prime}$ is non-hamiltonian, too. Consider $v \in V\left(G^{\prime}\right)$. As $G$ is almost hypohamiltonian, there exists a hamiltonian cycle in $G-v$, so there exists a hamiltonian path in $G^{\prime}-v$.

(ii) Since $H^{\prime}-w=H$ is non-traceable, $H^{\prime}-w$ and $H^{\prime}$ are non-hamiltonian. Let $v \in V\left(H^{\prime}\right) \backslash\{w\}$. As $H$ is a platypus, $H-v$ contains a hamiltonian path $\mathfrak{p}$ with end-vertices $v^{\prime}$ and $v^{\prime \prime}$. Now $\mathfrak{p} \cup v^{\prime} w v^{\prime \prime}$ is a hamiltonian cycle in $H^{\prime}-v$.

A non-hamiltonian graph $G$ is maximally non-hamiltonian if for every pair of non-adjacent vertices $v$ and $w$ in $G$, the graph $G+v w$ is hamiltonian.

Proposition 3.3. A maximally non-hamiltonian graph $G$ is a platypus if and only if $\Delta(G)<|V(G)|-1$.

Proof. Consider a maximally non-hamiltonian graph $G$ of order $n$. Assume $G$ contains a vertex $v$ of degree $n-1$. If $G$ is a platypus, then $G-v$ contains a hamiltonian path $\mathfrak{p}$. Let the end-vertices of $\mathfrak{p}$ be $x$ and $y$. But then $\mathfrak{p} \cup x v y$ is a hamiltonian cycle in $G$, a contradiction. ( $x v, v y \in E(G)$ since the degree of $v$ is $n-1$.)

Now let $G$ satisfy $\Delta(G)<n-1$. Consider $v \in V(G)$. We know that for $w \notin N[v]$, $G+v w$ is hamiltonian, ergo $G$ contains a hamiltonian path $\mathfrak{p}$ with end-vertices $v$ and $w$. Then $\mathfrak{p}-v$ is a hamiltonian path in $G-v$.

It is not difficult to construct for every $k \geq 1$ infinitely many $k$-connected maximally non-hamiltonian graphs. However, as far as the author is aware, for $k \geq 4$ all of these constructions have maximum degree $|V(G)|-1$, so the natural question whether 4-connected platypuses exist remains open at this point. For hypohamiltonian graphs it is a long-standing open problem whether 4-connected such graphs exist, see Thomassen's paper [34]. (The existence of 4-connected hypotraceable graphs is undecided as well.) We shall see in Section 6 that, in stark contrast to hypohamiltonian graphs, $k$-connected platypuses exist for every $k \geq 2$.

Consider graphs $G$ and $H$ containing cubic vertices $x \in V(G)$ and $y \in V(H)$. Then $G_{x} H_{y}$ is defined as one of the graphs obtained by taking $G-x$ and $H-y$, and identifying, using a bijection, $N(x)$ and $N(y)$. Thomassen [31] showed that if $G$ and $H$ are hypohamiltonian, then $G_{x} H_{y}$ is hypohamiltonian, too. In [43], we showed that if $G$ is hypohamiltonian and $H$ is almost hypohamiltonian, then $G_{x} H_{y}$ is almost hypohamiltonian, too (under the condition that $y$ is not the exceptional vertex of $H$ ). The next theorem is inspired by Thomassen's result mentioned above. Note that if a graph is hypohamiltonian, then each vertex of a triangle contained in that graph has degree at least 4 . Let $G$ be a graph and $e \in E(G)$. Then $G / e$ is the graph obtained by contracting $e$. 
Theorem 3.4. Let $G$ be a hypohamiltonian graph and $H$ a graph with cubic vertices $x \in V(G)$ and $y \in V(H)$, and put $N(x)=\left\{x_{1}, x_{2}, x_{3}\right\}$ and $N(y)=\left\{y_{1}, y_{2}, y_{3}\right\}$. Consider $G-x$ and $H-y$, and denote the graph obtained by identifying $x_{1}$ with $y_{1}$ and $x_{2}$ with $y_{2}$ by $\Gamma$. If $H$ is hypohamiltonian, then

(i) $\Gamma$ is a 2-leaf-stable graph and

(ii) $\Gamma+x_{3} y_{3}=\Gamma^{\prime}$ is a traceable platypus.

(iii) If $H$ is a platypus, then $\Gamma^{\prime} / x_{3} y_{3}=G_{x} H_{y}$ is a platypus.

Proof. In $\Gamma$, let $z_{1}$ and $z_{2}$ be the vertices obtained by identifying $x_{1}$ with $y_{1}$ and $x_{2}$ with $y_{2}$, respectively. We denote with $G_{x}$ and $H_{y}$ the copy of $G-x$ and $H-y$ in $\Gamma$, respectively. (The same nomenclature holds in $\Gamma^{\prime}$ and $G_{x} H_{y}$.)

(i) We show in (ii) that $\Gamma+x_{3} y_{3}$ is non-hamiltonian, whence, $\Gamma$ is non-hamiltonian and $\operatorname{ml}(\Gamma) \neq 1$. We now prove that $\Gamma-v$ is traceable, i.e. $\operatorname{ml}(\Gamma-v) \leq 2$, for all $v \in V(\Gamma)$. W.l.o.g. $v \in V\left(G_{x}\right)$. First, we treat the case $v \notin\left\{z_{1}, z_{2}\right\}$. Let $\mathfrak{g}$ be a hamiltonian cycle in $G$ avoiding the copy of $v$ in $G$. Put $\mathfrak{p}=\mathfrak{g}-x$. W.l.o.g. one of the end-vertices of $\mathfrak{p}$ is $z_{1}$. Let $\mathfrak{h}$ be a hamiltonian cycle in $H-y_{2}$. Since we can treat $\mathfrak{p}$ and $\mathfrak{h}-y=\mathfrak{q}$ as paths in $G_{x}$ and $H_{y}$, respectively, and as $\mathfrak{q}$ has $z_{1}$ as an end-vertex, $\mathfrak{p} \cup \mathfrak{q}$ is a hamiltonian path in $\Gamma-v$.

Now assume that $v \in\left\{z_{1}, z_{2}\right\}$. W.l.o.g. $v=z_{1}$. Combining the path obtained by deleting from a hamiltonian cycle of $G-x_{1}$ the vertex $x$ and the path obtained by deleting from a hamiltonian cycle of $H-y_{1}$ the vertex $y$ yields the traceability of $\Gamma-z_{1}$.

Suppose there exists a vertex $v$ such that $\Gamma-v$ contains a hamiltonian cycle $\mathfrak{h}^{\prime}$. Obviously, $v \notin\left\{z_{1}, z_{2}\right\}$. W.l.o.g. $v \in V\left(G_{x}\right)$. Then $\left(\mathfrak{h}^{\prime} \cap H_{y}\right) \cup z_{1} y z_{2}$ corresponds to a hamiltonian cycle in $H$, which is absurd. We have shown that $\Gamma-v$ is nonhamiltonian, i.e. that $\operatorname{ml}(\Gamma-v) \neq 1$ for every $v \in V(\Gamma)$. For $\Gamma$ to be 2-leaf-stable, it remains to show that $\Gamma$ is traceable - we do so in (ii).

(ii) Let $\Gamma^{\prime}$ contain a hamiltonian cycle $\mathfrak{h}$. Thomassen [31] showed that $\Gamma^{\prime} / x_{3} y_{3}=$ $G_{x} H_{y}$ is hypohamiltonian, ergo non-hamiltonian, so $\mathfrak{h}$ certainly does not contain $x_{3} y_{3}$. Hence, the path $\mathfrak{p}=\mathfrak{h} \cap G_{x}$ has end-vertices $z_{1}$ and $z_{2}$ and visits all vertices in $G_{x}$. Now consider $\mathfrak{p}$ to lie in $G$. But then $\mathfrak{p} \cup x_{1} x x_{2}$ is a hamiltonian cycle in $G$, a contradiction. So $\Gamma^{\prime}$ is non-hamiltonian. That $\Gamma^{\prime}-v$ is traceable for every $v \in V\left(\Gamma^{\prime}\right)$ follows directly from (i).

Combining the path obtained by deleting from a hamiltonian cycle of $G-x_{1}$ the vertex $x$ with the path obtained by deleting from a hamiltonian cycle in $H-y$ the edge $y_{2} w$, where $w \in N\left(y_{2}\right) \backslash\{y\}$, we obtain the traceability of $\Gamma^{\prime}$. As $x_{3} y_{3}$ is not an edge of this path, we have also shown that $\Gamma$ is traceable.

(iii) In $\Gamma^{\prime} / x_{3} y_{3}=G_{x} H_{y}$, let $z_{3}$ be the vertex obtained by identifying $x_{3}$ with $y_{3}$. Assume that $G_{x} H_{y}$ contains a hamiltonian cycle $\mathfrak{h}$. Consider $\mathfrak{h} \cap G_{x}$. If $\mathfrak{h} \cap G_{x}$ consists of two components, one of them is a single vertex. We denote the other component, which must contain at least two vertices, with $\mathfrak{p}$. If $\mathfrak{h} \cap G_{x}$ consists of one component, we call it $\mathfrak{p}$, as well. W.l.o.g. $\mathfrak{p}$ has end-vertices $z_{2}$ and $z_{3}$. If $z_{1} \in V(\mathfrak{p})$, and we consider for a moment $\mathfrak{p}$ to lie in $G-x$, then $\mathfrak{p} \cup z_{2} x z_{3}$ corresponds to a hamiltonian cycle in $G$ and we have a contradiction. If $z_{1} \notin V(\mathfrak{p})$, consider the path $\mathfrak{q}=\mathfrak{h} \cap H_{y}$ to lie in $H$. Now $\mathfrak{q} \cup y_{2} y y_{3}$ is a hamiltonian cycle in $H$, once again a contradiction. 
Consider $v \in V(H) \backslash N[y]$. Denote with $\mathfrak{p}^{\prime}$ a hamiltonian path in $H-v$. W.l.o.g. $y_{1} y \in E\left(\mathfrak{p}^{\prime}\right)$. Two cases arise: (a) $y$ is an end-vertex of $\mathfrak{p}^{\prime}$, or (b) it is not. We first treat case (a). Now clearly $y_{1}$ cannot be an end-vertex of $\mathfrak{p}^{\prime}$. Consider a hamiltonian cycle in $G-x_{2}$ and delete from it $x$. We obtain a path $\mathfrak{q}$ which visits all vertices in $G$ excluding $x$ and $x_{2}$, and has $x_{1}$ and $x_{3}$ as its end-vertices. Combining $\mathfrak{q}-x_{3}$ with $\mathfrak{p}^{\prime}-y$, we have shown that $G_{x} H_{y}-v$ is traceable. Concerning case (b), w.l.o.g. $y_{3} y \in E\left(\mathfrak{p}^{\prime}\right)$. Construct $\mathfrak{q}$ as in (a). Then $\mathfrak{q} \cup\left(\mathfrak{p}^{\prime}-y\right)$ corresponds to a hamiltonian path in $G_{x} H_{y}-v$.

Let $v \in V(G) \backslash N[x]$. Consider a hamiltonian cycle $\mathfrak{g}$ in $G-v$. W.l.o.g. $x_{1} x x_{2} \subset \mathfrak{g}$. Then the path $\mathfrak{g}-x$ has end-vertices $x_{1}$ and $x_{2}$. Let $\mathfrak{q}^{\prime \prime}$ be a hamiltonian path in $H-y_{3}$. Then combining $\mathfrak{q}^{\prime \prime}-y$ (which consists of one or two components) with $\mathfrak{g}-x$ yields a hamiltonian path (or hamiltonian cycle) in $G_{x} H_{y}-v$. (The treatment is very similar if $y_{2}$ or $y$ are an end-vertex of $\mathfrak{q}^{\prime \prime}$.)

Consider a hamiltonian cycle $\mathfrak{g}^{\prime}$ in $G-x_{1}$, and let $\mathfrak{p}^{\prime \prime}$ be a hamiltonian path in $H-y_{1}$. Putting $\mathfrak{g}^{\prime}-x$ and $\mathfrak{p}^{\prime \prime}-y$ together gives a hamiltonian path (or hamiltonian cycle) in $G_{x} H_{y}-z_{1}$ if $y$ is not an end-vertex of $\mathfrak{p}^{\prime \prime}$. Now assume that $y$ is an endvertex of $\mathfrak{p}^{\prime \prime}$ and let w.l.o.g. $y_{3} y \in E\left(\mathfrak{p}^{\prime \prime}\right)$. Removing from $\left(\mathfrak{g}^{\prime}-x\right) \cup\left(\mathfrak{p}^{\prime \prime}-y\right)$ an edge $e$ of $\mathfrak{p}^{\prime \prime}$ incident with $y_{2}$ such that $\left(\left(\mathfrak{g}^{\prime}-x\right) \cup\left(\mathfrak{p}^{\prime \prime}-y\right)\right)-e$ is connected yields a hamiltonian path in $G_{x} H_{y}-z_{1}$. For $z_{2}$ and $z_{3}$ the treatment is analogous.

By saying that "we add vertices $x_{1}, x_{2}, \ldots, x_{n}$ on an edge $x y$ " we mean that we replace the path isomorphic to $K_{2}$ having vertices $x, y$ by the path $x x_{1} x_{2} \ldots x_{n} y$ isomorphic to $P_{n+2}$.

Proposition 3.5. Let $G$ be a hypohamiltonian graph and $e=v_{1} v_{2} \in E(G)$. By adding vertices $v$ and $v^{\prime}$ on e, we obtain a platypus $G^{\prime}$.

Proof. W.l.o.g. assume that $v \in N\left(v_{1}\right)$ in $G^{\prime}$. As $G$ is non-hamiltonian, $G^{\prime}$ is nonhamiltonian, too. For every vertex $u \in V(G) \backslash\left\{v, v^{\prime}, v_{1}, v_{2}\right\}$ we have that $G-u$ contains a hamiltonian cycle $\mathfrak{h}$. h must contain some edge $e^{\prime}$ incident to $v_{1}$. If $e^{\prime}=e$, we are done. If not, then $\left(\mathfrak{h}-e^{\prime}\right) \cup v_{1} v v^{\prime}$ is a hamiltonian path in $G^{\prime}-u$.

Let $\mathfrak{h}^{\prime}$ be a hamiltonian cycle in $G-v_{1}$ and $w \in V(G)$ such that $w v_{2} \in E\left(\mathfrak{h}^{\prime}\right)$. Then $\left(\mathfrak{h}^{\prime}-w v_{2}\right) \cup v_{2} v^{\prime} v$ is a hamiltonian path in $G^{\prime}-v_{1}$. In the same way it can be shown that $G^{\prime}-v_{2}$ is traceable. Consider $w^{\prime} w^{\prime \prime} \in E\left(\mathfrak{h}^{\prime}\right)$, where $w^{\prime} \in N\left(v_{1}\right)$. Then $\left(\mathfrak{h}^{\prime}-w^{\prime} w^{\prime \prime}\right) \cup w^{\prime} v_{1} v$ is a hamiltonian path in $G^{\prime}-v^{\prime}$. Due to a similar argument $G^{\prime}-v$ is traceable.

By Prop. 2.1 (vii), Prop. 3.5 holds as well if only a single vertex is added on the edge $e$.

A graph $G$ is called homogeneously traceable if every vertex of the graph is an end-vertex of a hamiltonian path. It is easy to see that the following holds.

Proposition 3.6. Every non-hamiltonian homogeneously traceable graph is a platypus.

We now settle the open problem of Wiener [40] whether planar leaf-stable graphs without cubic vertices exist. Consider the dotted prism $\dot{C}_{k}$ over $C_{k}$ for $k \geq 3$ odd, and replace in each such graph each 3 -ear with a 4 -ear. Now take in each graph the 
end-vertices of each 4-ear and join them by an edge. It is clear that these graphs are planar and have no cubic vertices - we leave to the reader the straightforward verification that each of them is 2-leaf-stable. We have obtained the following.

Theorem 3.7. There exist infinitely many planar 2-leaf-stable graphs which have no cubic vertices.

Wiener also poses a meta-question: are there leaf-stable or leaf-critical graphs not based on hypohamiltonian graphs, in the sense that their construction does not use hypohamiltonian graphs as building blocks? With Theorem 3.7, we have answered this question positively.

In his paper [40], Wiener also asks for the smallest $\ell$-leaf-stable graphs, especially under the added condition of planarity. Consider $\mathfrak{T}$, the graph from Fig. 1 (a), and replace each 3-ear with a 4-ear. We call this newly obtained graph $\mathfrak{T}^{\prime}$. For the proof of the following statement, which settles Wiener's question for $\ell=2$, Van Cleemput and the author used a computer. (We exclude the graph $K_{2}$ from the discussion.)

Theorem 3.8. (Van Cleemput and Zamfirescu) $\mathfrak{T}^{\prime}$, a planar graph, is the smallest 2-leaf-stable graph, both in terms of order and size.

\section{Platypuses of connectivity 2}

Consider $G \in \mathcal{P}_{2}$, and let $X=\{x, y\}$ be a cut in $G$. We denote the pair $(G, X)$ with $G_{X}$. By Theorem 2.3, $G-X$ consists of exactly two connected components, say $A$ and $B$. Put $G_{X}^{\prime}=G[V(A) \cup\{x, y\}]$ and $G_{X}^{\prime \prime}=G[V(B) \cup\{x, y\}]$. We call $G_{X}^{\prime}$ and $G_{X}^{\prime \prime}$ halves of $G$ with respect to $X$, while $x$ and $y$ will be called the ends of $G_{X}^{\prime}$ and $G_{X}^{\prime \prime}$. (In Thomassen's language [33], $G_{X}^{\prime}$ and $G_{X}^{\prime \prime}$ are 2-fragments of $G$, while $x$ and $y$ are vertices of attachment of $A$ and $B$.) When we simply speak of a "half" we are referring to a half of an arbitrary $G \in \mathcal{P}_{2}$ w.r.t. an arbitrary (but fixed) 2-cut in $G$. A half $H$ is traversable if there exists a path between its ends which visits all vertices in $H$. Not both halves of $G$ w.r.t. $X$ can be traversable, so there are two cases:

(i) $G_{X}$ is semi-traversable if $G_{X}^{\prime}$ is non-traversable and $G_{X}^{\prime \prime}$ is traversable.

(ii) $G_{X}$ is non-traversable if neither $G_{X}^{\prime}$ nor $G_{X}^{\prime \prime}$ are traversable.

If, for all 2-cuts $X, G_{X}$ is semi-traversable (non-traversable), then we call $G$ semi-traversable (non-traversable). If a platypus of connectivity 2 is neither semitraversable nor non-traversable, we call it mixed-traversable. $\mathfrak{T}$ is an example of a semi-traversable platypus, and applying Theorem 3.4 (i) to two copies of the Petersen graph yields a mixed-traversable platypus. Every hypotraceable graph constructed using Thomassen's method introduced in [31] is a non-traversable platypus - an example of such a graph is given in [31, Fig. 3].

We now construct new platypuses from old ones by replacing halves. In order to do so, we need the following lemma, the proof of which is obvious.

Lemma 4.1. Let $G \in \mathcal{P}_{2}$ and $H$ be a half of $G$ with ends $x$ and $y$. Then there exists a path which spans $H-x$ and has $y$ as an end-vertex. 
Theorem 4.2. Let $G$ and $H$ be platypuses such that there are 2-cuts $X$ and $Y$ in $G$ and $H$, respectively. If

(i) $G_{X}$ is non-traversable, $H_{Y}^{\prime} \notin\left\{P_{3}, K_{3}\right\}, H_{Y}^{\prime}$ contains a hamiltonian path with $y \in Y$ as end-vertex, and $H_{Y}^{\prime \prime}$ is non-traversable, or

(ii) $G_{X}$ is semi-traversable with $G_{X}^{\prime}$ containing a hamiltonian path with $x \in X$ as end-vertex, $G_{X}^{\prime \prime} \notin\left\{P_{3}, K_{3}\right\}, G_{X}^{\prime \prime}$ is traversable, and $H_{Y}$ is semi-traversable with $H_{Y}^{\prime} \notin\left\{P_{3}, K_{3}\right\}$ and $H_{Y}^{\prime}$ traversable,

then by identifying (using a bijection) the ends of $G_{X}^{\prime}$ and $H_{Y}^{\prime}$ we obtain a platypus.

Proof. We denote with $\Gamma$ the resulting graph. Put $X=\left\{x_{1}, x_{2}\right\}$ and $Y=\left\{y_{1}, y_{2}\right\}$. For the following, we denote with $J_{G}$ and $J_{H}$ the copy of $G_{X}^{\prime}$ and $H_{Y}^{\prime}$ in $\Gamma$, respectively, and put $V\left(J_{G}\right) \cap V\left(J_{H}\right)=Z=\left\{z_{1}, z_{2}\right\}$, where the identification of $x_{i}$ and $y_{i}$ yields $z_{i}, i \in\{1,2\}$. Assume that $\Gamma$ contains a hamiltonian cycle $\mathfrak{h}$. Then $\mathfrak{h} \cap J_{G}$ is a path in $J_{G}$ between $z_{1}$ and $z_{2}$ visiting all vertices of $J_{G}$. This implies that $G_{X}^{\prime}$ is traversable, a contradiction. So $\Gamma$ is non-hamiltonian.

For $v \in Z$, the traceability of $\Gamma-v$ follows directly from Lemma 4.1.

(i) Let $v \in V\left(G_{X}^{\prime}\right) \backslash X$. Consider the intersection of a hamiltonian path in $G-v$ with $G_{X}^{\prime}$, and denote it with $\mathfrak{p}$. Clearly $\mathfrak{p}$ has either one or two components. If $\mathfrak{p}$ has two components, both cannot consist of only one vertex simultaneously, since $G_{X}^{\prime}$ is non-traversable, so $G_{X}^{\prime} \notin\left\{P_{3}, K_{3}\right\}$. There are two cases. (a) If both components contain more than one vertex, then $G_{X}^{\prime \prime}$ must be traversable, a contradiction. (b) If one of the two components consists of one vertex, w.l.o.g. $x_{1}$, then we obtain the desired hamiltonian path in $\Gamma-v$ by using the fact that $H_{Y}^{\prime}$ contains a hamiltonian path with $y_{2}$ as end-vertex. Now consider $\mathfrak{p}$ to lie in $J_{G}$ and let $\mathfrak{p}$ have one component. Since $G_{X}^{\prime \prime}$ is non-traversable, it neither is $P_{3}$ nor $K_{3}$, so $z_{1}$ or $z_{2}$ (possibly both) is (are) an end-vertex of $\mathfrak{p}$ - call this fact $(\star)$. Since $\mathfrak{p}$ visits all vertices in $J_{G}$ with the exception of $v$, Lemma 4.1 yields a hamiltonian path in $\Gamma-v$.

Let $v \in V\left(H_{Y}^{\prime}\right) \backslash Y$. Since $H_{Y}^{\prime} \notin\left\{P_{3}, K_{3}\right\}$ (this is needed in order to use $(\star)$ ) and $H_{Y}^{\prime \prime}$ is non-traversable, the arguments are the same as above.

(ii) Let $v \in V\left(G_{X}^{\prime}\right) \backslash X$. Consider the intersection of a hamiltonian path in $G-v$ with $G_{X}^{\prime}$, and denote it with $\mathfrak{p}$. Consider $\mathfrak{p}$ to lie in $J_{G}$. Let $\mathfrak{p}$ have two components. Then a hamiltonian path in $\Gamma-v$ is obtained by using a path between $z_{1}$ and $z_{2}$ visiting all vertices in $J_{H}$. Let $\mathfrak{p}$ have one component. Since $G_{X}^{\prime \prime} \notin\left\{P_{3}, K_{3}\right\},(\star)$ holds. Now Lemma 4.1 yields a hamiltonian path in $\Gamma-v$.

Let $v \in V\left(H_{Y}^{\prime}\right) \backslash Y$. Consider the intersection of a hamiltonian path in $H-v$ with $H_{Y}^{\prime}$, and denote it with $\mathfrak{q}$. If $\mathfrak{q}$ consists of two components, since $H_{Y}^{\prime \prime}$ is nontraversable, there are two possibilities. (a) Each component is a single vertex. This is the case if and only if $H_{Y}^{\prime} \in\left\{P_{3}, K_{3}\right\}$, but this was excluded in the theorem's statement. (b) One component is a single vertex, w.l.o.g. $z_{1}$, and one component contains more than one vertex. Since $J_{G}$ contains a hamiltonian path with $z_{2}$ as end-vertex, we obtain a hamiltonian path in $\Gamma-v$. Now let $\mathfrak{q}$ be connected. $(\star)$ holds, so we can use Lemma 4.1.

Thomassen asked in [34] whether hypohamiltonian graphs with minimum degree at least 4 exist. At the Cycles and Colourings conference in 2015, the author was 
asked by Tomáš Madaras the corresponding question for platypuses. After a solution to Madaras' problem was given by the author, Gunnar Brinkmann asked the same question, but where 4 is replaced with 5 . We can answer these questions positively.

\section{Theorem 4.3.}

(i) There exist infinitely many planar platypuses with minimum degree $\ell$ for every $\ell \in\{2,3,4,5\}$.

(ii) For every $d \geq 2$ there exists a platypus with minimum degree $d$.

Proof. Consider the dotted prism $\dot{C}_{k}$ over $C_{k}$ for $k \geq 3$ odd. Replace in each such graph each 3-ear with a 4-ear. This yields platypuses due to Lemma 2.6. We denote the family we obtain with $\mathcal{C}$.

(i) $\left\{\dot{C}_{k}\right\}_{k \geq 3}$ odd is an infinite family of planar platypuses with minimum degree 2 . It was shown by Thomassen that there exist infinitely many planar hypohamiltonian graphs [33], and that planar hypohamiltonian graphs have minimum degree 3 [34], so there exist infinitely many planar platypuses with minimum degree 3 . Now replace in each member of $\mathcal{C}$ each 4-ear having end-vertices $v, w$ with $(G, v, w)$, where $G$ is defined in Fig. 3 (a).

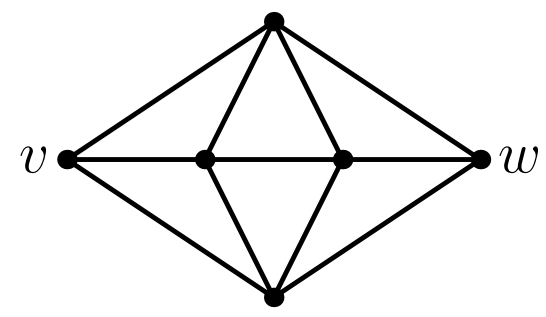

(a)

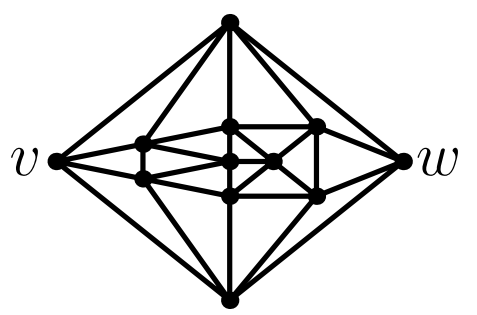

(b)

Fig. 3

The resulting graphs are platypuses due to Theorem 4.2 (ii). (We leave to the reader the verification that all requirements of 4.2 (ii) are met.) We have obtained an infinite family of planar platypuses with minimum degree 4 . Finally, in each member of $\mathcal{C}$, replace each 4-ear having end-vertices $v, w$ with $\left(G^{\prime}, v, w\right)$, where $G^{\prime}$ is defined in Fig. 3 (b). The resulting graphs are platypuses due to Theorem 4.2 (ii), and it is obvious that each member is planar and has minimum degree 5 .

(ii) Consider $\mathfrak{T}^{\prime}$, the platypus from Theorem 3.8. In $\mathfrak{T}^{\prime}$, replace each 4-ear with the complete graph $\left(K_{p}, v, w\right), p \geq 4$, where any choice of $v, w \in V\left(K_{p}\right)$ will do. We obtain a platypus due to Theorem 4.2 (ii). It has minimum degree $p-1$. For minimum degree 2 , consider $\mathfrak{T}$.

Theorem 4.3 (i) is complete in the sense that for no other values of $\ell$ the statement would be true, since there are no planar graphs with minimum degree at least 6 . We shall see in Section 6 that, with more tools, Theorem 4.3 (ii) can be improved dramatically. 


\section{$5 \quad$ Platypuses of connectivity 3}

By Corollary 2.7, there are no 3-connected platypuses on fewer than ten vertices. Of course, a 3-connected platypus has minimum degree at least 3. We have the following.

Theorem 5.1. The Petersen graph is among 3-connected platypuses of both minimum order and minimum size.

Recall that every hypohamiltonian graph is a platypus, and that every hypohamiltonian graph is 3-connected. We summarize two important results from [21].

Theorem 5.2 (Jooyandeh, McKay, Östergård, Pettersson, and Zamfirescu [21]). There exists a planar hypohamiltonian graph of order 40 and of order $n$ for every $n \geq 42$.

It turns out that the smallest polyhedral (i.e. planar and 3-connected) platypus has order significantly smaller than 40 . The smallest known such graph is presented in the following. To shorten the next proof, we require a useful result of Grinberg.

Grinberg's Criterion [13]. Given a plane graph with a hamiltonian cycle $\mathfrak{h}$ and exactly $f_{i}\left(f_{i}^{\prime}\right) i$-gons inside (outside) of $\mathfrak{h}$, we have

$$
\sum_{i \geq 3}(i-2)\left(f_{i}-f_{i}^{\prime}\right)=0
$$

Theorem 5.3. There exists a polyhedral platypus of order $n$ for every $n \geq 25$.

Proof. Let $\mathfrak{L}$ be the graph shown in Fig. 4. By Grinberg's Criterion, $\mathfrak{L}$ is nonhamiltonian: $(\dagger)$ becomes $\pm 1+3\left(f_{5}-f_{5}^{\prime}\right)=0$, which is impossible. Now we prove that for every $v \in V(\mathfrak{L})$, the graph $\mathfrak{L}-v$ is indeed traceable, where using "..." between two letters means a sequence of letters in their alphabetical order (possibly backwards).

b:ac...y. $\quad \mathbf{c}: a b n \ldots d o \ldots y . \quad \mathbf{d}: a b c g f e q \ldots h t s r y \ldots u . \quad$ e: $i \ldots n b a c d o \ldots s f g h t u y x w v$.

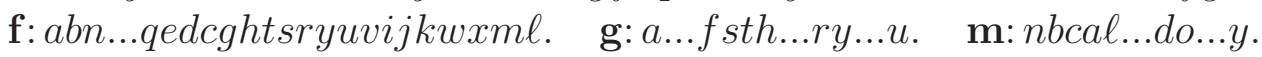

$\mathbf{n}: y x m \ell a \ldots d o p q e . . . k w \ldots r . \quad \mathbf{o}: w \ldots s f \ldots n b a c d e q p x y r . \quad \mathbf{p}: a \ldots d o \ldots e q \ldots y$.

$\mathbf{q}: a \ldots p x \ldots r y . \quad \mathbf{r}: a b n \ldots q e d c g f s t h \ldots m x \ldots u y . \quad \mathbf{s}: a \ldots r y \ldots t$.

$\mathbf{x}$ : abn...kwvijcdopqe...htuyrs. $\mathbf{y}: a \ldots x$.

Put $T^{0}(\mathfrak{L})=\mathfrak{L}$. The infinite family

$$
\left\{T^{k}(\mathfrak{L})\right\}_{k \geq 0} \cup\left\{R\left(T^{k}(\mathfrak{L})\right)\right\}_{k \geq 0} \cup\left\{S\left(T^{k}(\mathfrak{L})\right)\right\}_{k \geq 0}
$$

yields the statement, where $T, R$, and $S$ are defined in Prop. 2.2. 


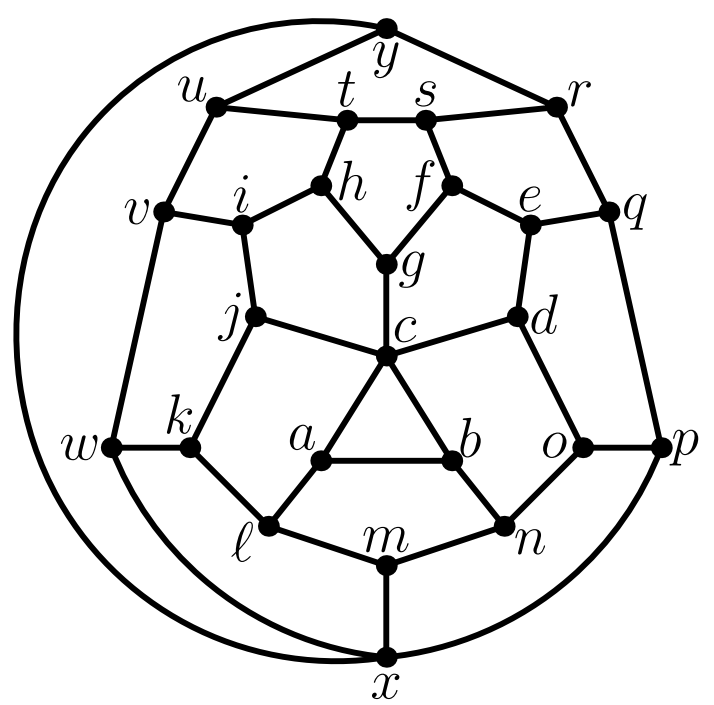

Fig. 4: $\mathfrak{L}$, a polyhedral platypus on 25 vertices

\section{Platypuses of higher connectivity}

One of the central problems concerning the theory of hypohamiltonian graphs is Thomassen's question from 1978 whether 4-connected such graphs exist [34]. (Such a graph cannot be planar due to a famous theorem of Tutte [36].) It is even unknown whether hypohamiltonian graphs with minimum degree at least 4 exist, a problem also posed by Thomassen [34]. In Section 4, we settled the corresponding question for platypuses. In [43], the author shows that there exist infinitely many almost hypohamiltonian graphs which are 4-connected. (But whether 5-connected such graphs exist is unknown.) If we relax Thomassen's question and ask for 4-connected platypuses, we realize that in fact much more can be shown.

Theorem 6.1. There exists a $k$-connected platypus for every $k \geq 2$, and a $k^{\prime}$-regular $k^{\prime}$-connected platypus for every $k^{\prime} \geq 3$.

Proof. In Section 4 we discussed platypuses of connectivity 2. For the remaining cases, our main tool will be a method of Meredith [25]. We briefly repeat his construction. Label Petersen's graph $P$ as in Fig. 5 and let $H_{k}$ be the loopless multigraph (i.e. a graph admitting multiple edges between two vertices) obtained from it as follows; replace each edge $A_{i} B_{i}$ with $b$ edges, $1 \leq i \leq 5$, and each of the other edges with $a$ edges, where if $k=3 \ell+a, a \in\{-1,0,1\}$, then $a=\ell, b=\ell+a a$.

$G_{k}$ is the (necessarily unique) graph obtained by expanding in $H_{k}$ each vertex to a $K_{k, k-1}$. We explain how this is done concretely, noting that in each vertex of the Petersen graph (see Fig. 5) the replacement is performed in the same manner. Denote the vertices of the copy of $K_{k, k-1}$ replacing $A_{1}$ with $a_{1}, \ldots, a_{k}, b_{1}, \ldots, b_{k-1}$, where $a_{i} a_{j} \notin E\left(G_{k}\right)$ and $b_{i} b_{j} \notin E\left(G_{k}\right)$ for all $i, j$. Then $a_{1}, \ldots, a_{\left\lfloor\frac{k-1}{2}\right\rfloor}\left(a_{\left\lceil\frac{k+3}{2}\right\rceil}, \ldots, a_{k}\right)$ are each joined by an edge to a corresponding vertex of the copy of $K_{k, k-1}$ replacing $A_{5}\left(A_{2}\right)$, while every vertex $a_{i}$, where $\left\lfloor\frac{k-1}{2}\right\rfloor<i<\left\lceil\frac{k+3}{2}\right\rceil$, is joined by an edge to a corresponding vertex in the copy of $K_{k, k-1}$ replacing $B_{1}$. (For more details, please see [25].) Meredith shows that for $k \geq 3, G_{k}$ is $k$-regular, $k$-connected, and non-hamiltonian. 


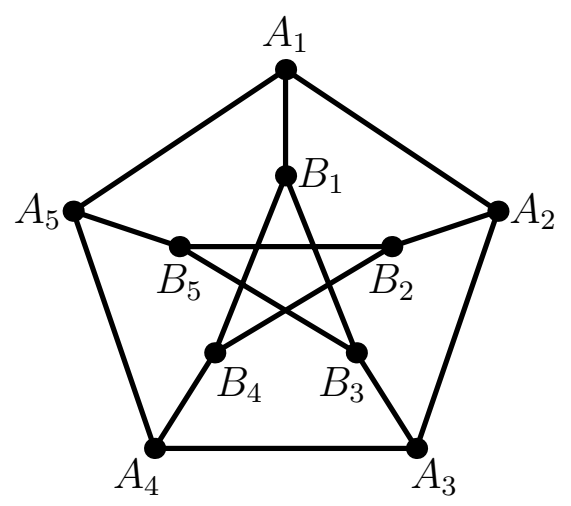

Fig. 5

It remains to prove that every vertex-deleted subgraph of $G_{k}$ is traceable. Consider $v \in V(P)$. There exists a hamiltonian cycle in $P-v$. This yields a cycle $\mathfrak{c}$ of length $n-(2 k-1)$ in $G_{k}$, where $n$ is the order of $G_{k}$, since exactly one $K_{k, k-1}$ is avoided by $\mathfrak{c}$, namely the one corresponding to the vertex $v$. We denote the vertices of this avoided complete bipartite graph with $a_{1}, \ldots, a_{k}, b_{1}, \ldots, b_{k-1}$, where $a_{i} a_{j} \notin E\left(G_{k}\right)$ and $b_{i} b_{j} \notin E\left(G_{k}\right)$ for all $i, j$. In $G_{k}$, let $x \in N\left(a_{1}\right) \backslash\left\{b_{i}\right\}_{i=1}^{k-1}$. Consider $x y \in E(\mathfrak{c})$ and put $\mathfrak{c}^{\prime}=\mathfrak{c}-x y$. Now $\mathfrak{c}^{\prime \prime}=\mathfrak{c}^{\prime} \cup x a_{1} b_{1} a_{2} b_{2} \ldots a_{k-1} b_{k-1}$ is a hamiltonian path in $G_{k}-a_{k}$ with end-vertices $y$ and $b_{k-1}$, and $\left(\mathfrak{c}^{\prime \prime} \cup b_{k-1} a_{k}\right)-y$ is a hamiltonian path in $G_{k}-y$. All other vertices in $G_{k}$ behave similarly.

By a theorem of Petersen [27], every cubic bridgeless graph contains a perfect matching. There exist infinitely many cubic hypohamiltonian graphs - consider for instance the so-called generalized Petersen graphs $G(n, k)$, originally defined by Coxeter [7] but baptized by Watkins [39]. Now $G(n, 2)$ with $n=5$ (mod. 6 ) is hypohamiltonian. (Robertson showed their non-hamiltonicity [28].) In this situation, above approach can be adapted to prove that for every $k \geq 3$ there exist infinitely many $k$-connected platypuses.

\section{Discussion}

1. Prop. 2.1 (vii) states that if a platypus contains a 4-ear, then this 4-ear can be replaced with a 3-ear, and the resulting graph is a platypus, as well. A priori there does not seem to be an argument that vice versa this must hold-but all examples encountered, for instance the family discussed in Theorem 2.4 and Corollary 2.7, allowed this. So we ask: Is there a platypus containing a 3-ear, which if replaced with a 4-ear, yields a graph that is not a platypus?

2. In the light of Theorem 2.9 we ask for an upper bound on the size of a platypus. We can show that for every $n \geq 9$ there exists a platypus of order $n$ and size $\left(\begin{array}{c}n-6 \\ 2\end{array}\right)+12$. Following [3], the size of a 2-connected non-hamiltonian graph on at least ten vertices is at most $\left(\begin{array}{c}n-2 \\ 2\end{array}\right)+4$.

3. In Section 3, we proposed the study of "full" platypuses, i.e. platypuses $G$ for which for every pair of non-adjacent vertices $v, w \in V(G)$, the graph $G+v w$ is not a platypus. (In other words, we are asking for maximally non-hamiltonian platypuses.) 
An example of such a well-fed platypus is the graph we obtain if we add in $\mathfrak{T}$ to each 3 -ear an edge between its end-vertices. The fact that this is indeed a full platypus follows from Corollary 2.7.

4. Although $\mathfrak{L}$, the graph from Fig. 4, is the smallest known polyhedral platypus, it does not address the question of the existence of a small polyhedral $\ell$-leaf-critical or $\ell$-leaf-stable graph, as $\operatorname{ml}(\mathfrak{L}-a) \neq 1$ (since for a hamiltonian cycle $\mathfrak{h}$ in $\mathfrak{L}-a$ we would have $b c \in E(\mathfrak{h})$, so then $(\mathfrak{h}-b c) \cup b a c$ would be a hamiltonian cycle in $\mathfrak{L}$, a contradiction), so $\operatorname{ml}(\mathfrak{L}-a)=2$, but $\operatorname{ml}(\mathfrak{L}-y)=1$. Since among leaf-stable and leaf-critical graphs, only the families of 2-leaf-stable and $\{2,3\}$-leaf-critical graphs are contained in the class of platypuses, we focus thereon. The smallest known polyhedral 2-leaf-critical (i.e. hypohamiltonian) graphs have 40 vertices [21]. Thomassen was the first to show that polyhedral 3-leaf-critical (i.e. hypotraceable) graphs exist [33]. His smallest example had 515 vertices. Currently, the smallest known such graph has order 190. (This number is obtained by applying Thomassen's method from [33] to a 40-vertex planar hypohamiltonian graph [21].) The smallest known polyhedral 2-leaf-stable graph is due to Wiener and has order 152, see [40], and is also based on the smallest known planar hypohamiltonian graph [21].

5. Let $\psi_{k}\left(\bar{\psi}_{k}\right)$ be the order of the smallest platypus (smallest planar platypus) of connectivity $k$. We showed that

$$
\psi_{2}=\bar{\psi}_{2}=9, \quad \psi_{3}=10, \quad \bar{\psi}_{3} \leq 25, \quad \text { and } \quad \psi_{k} \leq 20 k-10 .
$$

We ask here for improving these bounds or showing their optimality, especially in the cases of $\bar{\psi}_{3}$ and $\psi_{4}$.

Acknowledgements. The author is supported by a Postdoctoral Fellowship of the Research Foundation Flanders (FWO). I am grateful to the anonymous referees for their helpful comments. I also thank Nico Van Cleemput for his work to find smallest examples in various families of graphs, Kenta Ozeki for the initial idea, and Gunnar Brinkmann, Tomáš Madaras, Iulia Mihai, and Tudor Zamfirescu for valuable feedback.

\section{References}

[1] M. Araya and G. Wiener. On Cubic Planar Hypohamiltonian and Hypotraceable Graphs. Electron. J. Combin. 18 (2011) \#P85.

[2] G. Brinkmann and N. Van Cleemput. Personal communication.

[3] O. D. Byer and D. L. Smeltzer. Edge bounds in nonhamiltonian $k$-connected graphs. Discrete Math. 307 (2007) 1572-1579.

[4] V. Chvátal. Flip-flops in hypohamiltonian graphs. Canad. Math. Bull. 16 (1973) $33-41$.

[5] V. Chvátal. New directions in hamiltonian graph theory. In: New Directions in the Theory of Graphs (ed.: F. Harary), Academic Press, New York (1973) 65-95.

[6] V. Chvátal. Tough graphs and hamiltonian circuits. Discrete Math. 5 (1973) 215-228. 
[7] H. S. M. Coxeter. Self-dual configurations and regular graphs. Bull. Amer. Math. Soc. 56 (1950) 413-455.

[8] S. Fiorini. Hypohamiltonian snarks. In: Graphs and Other Combinatorial Topics, Proc. 3rd Czechoslovak Symp. on Graph Theory, Prague, Aug. 24-7, 1982 (ed.: M. Fiedler), Teubner-Texte zur Math., Bd. 59, Teubner, Leipzig (1983) 70-75.

[9] T. Gallai. Problem 4. In: Theory of Graphs, Proc. Tihany 1966 (eds.: P. Erdős \& G. Katona), Academic Press, New York (1968), p. 362.

[10] L. Gargano, M. Hammar, P. Hell, L. Stacho, and U. Vaccaro. Spanning spiders and light-splitting switches. Discrete Math. 285 (2004) 83-95.

[11] J. Goedgebeur and C. T. Zamfirescu. Infinitely many planar cubic hypohamiltonian graphs of girth 5. Submitted.

[12] J. Goedgebeur and C. T. Zamfirescu. On Hypohamiltonian Snarks and a Theorem of Fiorini. Submitted. arXiv:1608.07164 [math.CO]. To appear in: Ars Math. Contemp.

[13] E. J. Grinberg. Plane homogeneous graphs of degree three without Hamiltonian circuits. Latvian Math. Yearbook 4 (1968) 51-58. (Russian)

[14] S. Gutt. Infinite families of hypohamiltonian graphs. Acad. Roy. Belg. Bull. Cl. Sci. V. Sér. 63 (1977) 432-440.

[15] R. Halin. Zur Theorie der $n$-fach zusammenhängenden Graphen. Abh. Math. Sem. Hamburg 33 (1969) 133-164. (German)

[16] J. C. Herz. Sur la cyclabilité des graphes. Computers in Math. Research, NorthHolland (1968) 97-107. (French)

[17] D. A. Holton, B. D. McKay, M. D. Plummer, and C. Thomassen. Cycles through specified vertices in 3-connected graphs. Combinatorica 1 (1981) 409-418.

[18] D. A. Holton and J. Sheehan. The Petersen Graph, Chapter 7: Hypohamiltonian graphs, Cambridge University Press, New York, 1993.

[19] R. Isaacs. Infinite families of non-trivial trivalent graphs which are not Tait-colorable. Amer. Math. Monthly 82 (1975) 221-239.

[20] S. Jendrol' and Z. Skupień. Exact Numbers of Longest Cycles with Empty Intersection. Europ. J. Combin. 18 (1997) 575-578.

[21] M. Jooyandeh, B. D. McKay, P. R. J. Östergård, V. H. Pettersson, and C. T. Zamfirescu. Planar Hypohamiltonian Graphs on 40 Vertices. J. Graph Theory 84 (2017) 121-133.

[22] E. Máčajová and M. Škoviera. Constructing Hypohamiltonian Snarks with Cyclic Connectivity 5 and 6. Electron. J. Combin. 14 (2007) \# R18.

[23] E. Máčajová and M. Škoviera. Infinitely Many Hypohamiltonian Cubic Graphs of Girth 7. Graphs Combin. 27 (2011) 231-241.

[24] B. D. McKay. Hypohamiltonian Planar Cubic Graphs with Girth 5. J. Graph Theory, DOI: 10.1002/jgt.22043. arXiv:1507.07197 [math.CO]. 
[25] G. H. J. Meredith. Regular $n$-Valent $n$-Connected NonHamiltonian Non- $n$-EdgeColorable Graphs. J. Combin. Theory, Ser. B 14 (1973) 55-60.

[26] T. Nishizeki. A 1-tough nonhamiltonian maximal planar graph. Discrete Math. 30 (1980) 305-307.

[27] J. Petersen. Die Theorie der regulären Graphs. Acta Math. 15 (1891) 193-220. (German)

[28] G. N. Robertson. Graphs minimal under girth, valency and connectivity constraints. $\mathrm{Ph}$. D. thesis, Waterloo, Ontario, Canada (University of Waterloo, 1969).

[29] A. Shabbir, C. T. Zamfirescu, and T. I. Zamfirescu. Intersecting longest paths and longest cycles: A survey. Electron. J. Graph Theory Appl. 1 (2013) 56-76.

[30] P. G. Tait. Remarks on the Colourings of Maps. Proc. R. Soc. Edinburgh 10 (1880) 729.

[31] C. Thomassen. Hypohamiltonian and hypotraceable graphs. Discrete Math. 9 (1974) 91-96.

[32] C. Thomassen. On hypohamiltonian graphs. Discrete Math. 10 (1974) 383-390.

[33] C. Thomassen. Planar and infinite hypohamiltonian and hypotraceable graphs. Discrete Math. 14 (1976) 377-389.

[34] C. Thomassen. Hypohamiltonian graphs and digraphs. Theory and Applications of Graphs, Lecture Notes in Mathematics 642, Springer, Berlin (1978) 557-571.

[35] C. Thomassen. Planar cubic hypohamiltonian and hypotraceable graphs. J. Combin. Theory, Ser. B 30 (1981) 36-44.

[36] W. T. Tutte. A theorem on planar graphs. Trans. Amer. Math. Soc. 82 (1956) 99-116.

[37] N. Van Cleemput. Personal communication.

[38] H. Walther. Über die Nichtexistenz eines Knotenpunktes, durch den alle längsten Wege eines Graphen gehen. J. Combin. Theory 6 (1969) 1-6. (German)

[39] M. E. Watkins. A Theorem on Tait Colorings with an Application to the Generalized Petersen Graphs. J. Combin. Theory 6 (1969) 152-164.

[40] G. Wiener. Leaf-Critical and Leaf-Stable Graphs. J. Graph Theory 84 (2017) 443459.

[41] G. Wiener and M. Araya. On planar hypohamiltonian graphs. J. Graph Theory 67 (2011) 55-68.

[42] C. T. Zamfirescu. Hypohamiltonian graphs and their crossing number. Electron. J. Combin. 19 (2012) \#P12.

[43] C. T. Zamfirescu. On Hypohamiltonian and Almost Hypohamiltonian Graphs. J. Graph Theory 79 (2015) 63-81.

[44] C. T. Zamfirescu and T. I. Zamfirescu. A planar hypohamiltonian graph with 48 vertices. J. Graph Theory 55 (2007) 338-342. 